\title{
Variación genética en el complejo infraespecífico de chayote evaluada mediante sistemas isoenzimáticos
}

\author{
Carlos Hugo Avendaño-Arrazate ${ }^{(1)}$, Jorge Cadena-Iñiguez ${ }^{(2)}$, María Lourdes Arévalo-Galarza(3), \\ Victor Manuel Cisneros-Solano(4), Juan Francisco Aguirre-Medina( ${ }^{(1)}$, Esaú del Carmen Moreno-Pérez ${ }^{(4)}$, \\ Moises Cortés-Cruz ${ }^{(1)}$, Carlos Roman Castillo-Martínez ${ }^{(1)}$ y Porfirio Ramírez-Vallejo(3)
}

\begin{abstract}
(1)Instituto Nacional de Investigaciones Forestales Agrícolas y Pecuarias México, Apartado Postal 96, Km 18 Carretera Tapachula-Cacahoatán, Tuxtla Chico, CP 30870 Chiapas. México. E-mail: avendano.carlos@inifap.gob.mx, aguirre.juan@inifap.gob.mx, cortes.moises@inifap.gob.mx, castillo.carlos@inifap.gob.mx ${ }^{(2)}$ Colegio de Postgraduados (CPG), Campus San Luis Potosí, Calle Iturbide n 73, CP 78600 Salinas de Hidalgo, S.L.P., México. E-mail: jocadena@colpos.mx ${ }^{(3)}$ CPG, Km 36,5 Carretera México-Texcoco CP 56230 Texcoco, Estado de México, México. E-mail: larevalo@colpos.mx, pramirez@colpos.mx ${ }^{(4)}$ Universidad Autónoma Chapingo, Km. 38,5 Carretera México-Texcoco, CP 56230 Texcoco, Estado de México, México. E-mail: esaump10@yahoo.com.mx
\end{abstract}

Resumen - El objetivo de este trabajo fue estimar el grado de variación genética dentro del complejo infraespecífico de Sechium mediante el uso de sistemas isoenzimáticos. Se analizaron 23 loci codificados por 12 sistemas isoenzimáticos, en geles de almidón, en 10 individuos de cada una de las 30 accesiones (27 cultivadas y tres silvestres). La variación genética se estimó con base en el número promedio de alelos por locus (NPAL), porcentaje de porlimorfismo (PP), heterocigosidad observada y esperada (Ho y He), índice relativo de heterocigosidad (IRH) e índice de Shannon (IS). Para NPAL y PP, el promedio para las 30 accesiones fue de 2,03 y 59,8\%, respectivamente. El análisis de Ho y He mostró variación genética en el complejo infraespecífico de Sechium, con valores promedio de 0,05 y 0,26 , respectivamente. El IRH mostró una deficiencia de individuos heterocigotos (promedio de -0,75). El IS mostró gran diversidad en las 30 accesiones $(0,41)$. Las poblaciones con mayor diversidad fueron Negrito, Verde liso, Negro xalapa, Verde espinoso y Negro cónico; con una variación intermedia fueron Castilla blanco, Caldero y Blanco pequeño; y, con poca variación, Castilla verde, Cambray y los parientes silvestres.

Términos para indexación: Sechium, diversidad genética, estrategias de conservación, frecuencias alélicas, índice de heterocigosidad, mejoramiento genético.

\section{Genetic variation of an infraspecific chayote complex evaluated by isoenzimatic systems}

\begin{abstract}
The objective of this work was to estimate the degree of genetic variation in an infraspecific complex of Sechium using isoenzymatic systems. For this purpose, 23 loci were codified by 12 isoenzimatic systems and analyzed in starch gels, in 10 individuals from each of the 30 accesions of Sechium (27 cultivated and three wild relatives). The estimation of genetic variation was based on the average number of alleles per locus (NPAL), polymorphism percentage (PP), observed and expected heterozygosity (Ho and He), relative heterozygosity index (RHI) and Shannon's index (IS). For NPAL and PP, the average for the 30 accesions was 2.03 and 59.8\%, respectively. The analysis of Ho and He showed genetic variation in the infraspecific complex of Sechium, with 0.05 and 0.26 average values, respectively. The RHI showed a deficiency of heterozygote individuals (average of -0.75). The IS showed a great diversity in the 30 accesions (0.41). The populations with the greatest genetic variations were Negrito, Verde liso, Negro xalapa, Verde espinoso and Negro cónico; with intermediate variation were Castilla blanco, Caldero and Blanco pequeño; and with little variation were Castilla verde, Cambray and wild relatives.
\end{abstract}

Index terms: Sechium, genetic diversity, conservation strategies, allele frequencies, heterozygosity index, genetic breeding.

\section{Introducción}

México es el centro de diversidad de muchas especies cultivadas importantes, entre ellas la del chayote, Sechium edule (Jacq.) Sw., que pertenece a la familia Cucurbitaceae (Newstrom, 1991). La mayor diversidad del género Sechium se localiza en los estados de Veracruz, Chiapas y Oaxaca (Lara-Saade, 1996). Sobresale el complejo infraespecífico de $S$. edule como especie con amplia variación representada en México (Cadena-Iñiguez et al., 2007; Avendaño-Arrazate et al., 2010). Diferentes estudios han contribuido a tratar de explicar la variación registrada en este complejo, tanto en México como en Centroamérica y

Pesq. agropec. bras., Brasília, v.47, n.2, p.244-252, fev. 2012 
Sudamérica (Maffioli, 1981; Engels, 1983; Newstrom, 1991; Lara-Saade, 1996). Estos estudios han abordado principalmente la variación en fruto (tamaño, forma, color, sabor, presencia o ausencia de espinas), características de hojas y guías, así como respuesta de la especie a diferentes ambientes, con base en aspectos morfo-anatómicos, fisiológicos y bioquímicos (Cadena-Iñiguez et al., 2001; Cadena-Iñiguez, 2005).

Cadena-Iñiguez et al. (2008) han atribuido las variantes morfológicas observadas en algunos tipos de chayote a un proceso de especialización adaptativa ante los cambios ambientales. Abdelnour \& Rocha (2008), mediante el uso de marcadores isoenzimáticos, determinaron el nivel de diversidad genética en 42 accesiones de chayote de Costa Rica, y encontraron que 8 de 14 loci fueron polimórficos. Cadena-Iñiguez (2005) encontró, en 18 poblaciones de Sechium edule, un porcentaje promedio de polimorfismo de $40.6 \%$ mediante marcadores AFLP. En el género Cucurbita se han observado diferentes porcentajes de polimorfismo; Gwanama et al. (2000) encontraron 23,2\% de polimorfismo en Cucurbita moschata Duchesne, mediante marcadores RAPD; Katzir et al. (2000) usaron ISSR y registraron hasta un $80 \%$ de polimorfismo, en 28 accesiones de Cucurbita pepo L. El conocimiento de la variación fenotípica y genotípica, en el complejo infraespecífico del género Sechium, puede ser de gran utilidad en el diseño de métodos y estrategias de conservación y programas de mejoramiento genético del chayote.

El objetivo de este trabajo fue estimar el grado de variación genética dentro del complejo infraespecífico de Sechium mediante el uso de sistemas isoenzimáticos.

\section{Materiales y Métodos}

Se usaron frutos de 27 accesiones cultivadas y tres silvestres de S. edule, S. chinantlense (R. Lira \& F. Chiang) y S. compositum (Donn.Sm.) C. Jeffrey (Cuadro 1) del Banco de Germoplasma de Chayote, ubicado en Huastusco, Veracruz $\left(1^{\circ} 08^{\prime} 48^{\prime \prime N}\right.$, $97^{\circ} 57^{\prime} 00^{\prime \prime} \mathrm{W}$ ), de los cuales se consideraron las características principales y distintivas de las variedades (Figura 1).

Los análisis se hicieron con base en el protocolo derivado de Stubert \& Goodman (1988), en el laboratorio de marcadores moleculares del Colegio de Postgraduados, en Montecillo, Estado de México, México. El extracto isoenzimático se obtuvo a partir de radículas de nueve semillas de chayote de cada accesión. Debido a que las semillas, tanto de $S$. edule como de $S$. chinantlense y $S$. compositum, son del tipo endocárpica (Font, 1979), es decir, que germinan dentro del fruto (Flores, 1989), sin riesgo de fermentación, se usaron los frutos completos previamente lavados con agua destilada y tratados con el fungicida-insecticida Metacaptán - captan: Cis-N[(triclorometil) tio]-4-ciclohexen-1,2-dicarboximida al 65\%, más metoxicloro:2,2-bis(P-metoxifenil)- 1,1,1 -tricloroetano al 10\%), diluido en agua al 1\%.

La germinación se desarrolló en bandejas con agrolita como sustrato, en una cámara de crecimiento (modelo E 62 V.C.A. $127 \mathrm{H} 260$ ) a $26^{\circ} \mathrm{C}$ y completa oscuridad. Una muestra de $0,5 \mathrm{~g}$ de tejido de radículas de cada accesión fue colocada en un tubo Eppendorf de $1,5 \mu \mathrm{L}$, al cual se agregaron $100 \mu \mathrm{L}$ de solución amortiguadora de extracción con pH 7,5. El tejido se maceró con una punta de plástico, adaptada a un taladro eléctrico durante dos a tres segundos. Una vez terminada la maceración, el tejido (muestra de $14 \mathrm{~g}$ ) se mantuvo en hielo a $-4^{\circ} \mathrm{C} y$, posteriormente, se centrifugó (centrífuga modelo GS-15R) durante 20 min. El sobrenadante del centrifugado se colocó en tubos Eppendorf de 0,5 $\mu \mathrm{L}$, y los extractos finales se almacenaron a $-80^{\circ} \mathrm{C}$. Se realizó una prueba preliminar con 18 sistemas isoenzimáticos, de los cuales unicamente se evaluaron los 12 sistemas que presentaron polimorfismo entre loci, así como alelos por loci entre accesiones: catalasa-Cat (E.C.1.11.1.6), esterasa-Est (E.C.3.1.1), glutamato oxalacetato transaminasa-Got (E.C.2.6.1.2), isocitrato deshidrogenasa-Idh (E.C.1.1.1.42), malato deshidrogenasa-Mdh (E.C.1.1.1.37), enzima málica-Me (E.C.1.1.1.40), peroxidasa-Prx (E.C.1.11.1.7), 6-fosfogluconato deshidrogenasa-Pgd (E.C.1.1.1.44), fosfoglucomutasa-Pgm (E.C.2.7.5.1), ribulato bibosfato carboxilasa-Rubisco (E.C.4.1.1.39), shikimato deshidrogenasa-Sad (E.C.1.1.1.25) y fosfato isomerasa-Phi (E.C.5.1.3.9). La nomenclatura utilizada para cada alelo, en cada locus, se determinó por la abreviatura de las primeras letras de la enzima y por su distancia de migración, la cual varió de 90 (migración corta o lenta) a $115 \mathrm{~mm}$ (migración rápida o larga), a partir del inicio del corrimiento en el borde inferior del zimograma, cuya lectura se hizo visualizando patrones 
Cuadro 1. Accesiones de chayote usadas en el estudio isoenzimático.

\begin{tabular}{|c|c|c|c|c|c|}
\hline Accesión & Variedad & Localidad & Coordenadas geográficas LN-LW & Altitud (m) & Clave \\
\hline GISeM 286 & Cambray & Coscontla, Ver. & $19^{\circ} 08^{\prime} ; 95^{\circ} 59^{\prime}$ & 1.356 & $286 \mathrm{ca}$ \\
\hline GISeM 346 & Castilla blanco & Tlaltengo, Coscomatepec, Ver. & $19^{\circ} 05^{\prime} 00^{\prime \prime} ; 97^{\circ} 00^{\prime} 40^{\prime \prime}$ & 1.480 & $346 \mathrm{cb}$ \\
\hline GISeM 362 & Castilla blanco & Elotepec, Huatusco, Ver. & $19^{\circ} 09^{\prime} ; 95^{\circ} 58^{\prime}$ & 1.356 & $362 \mathrm{cb}$ \\
\hline GISeM 343 & Blanco pequeño & Tlaltengo, Coscomatepec, Ver. & $19^{\circ} 05^{\prime} 00^{\prime \prime} ; 97^{\circ} 00^{\prime} 40^{\prime \prime}$ & 1.480 & $343 b p$ \\
\hline GISeM 380 & Sechium edule & Tecolayo, Ixtaczoquitlán, Ver. & $18^{\circ} 48^{\prime} ; 97^{\circ} 01^{\prime}$ & 1.020 & 380 ses \\
\hline GISeM 292 & Negro cónico & Ixhuatlán del Café, Ver. & $19^{\circ} 05^{\prime} 00^{\prime \prime} ; 97^{\circ} 00^{\prime} 40^{\prime \prime}$ & 1.320 & $292 \mathrm{nc}$ \\
\hline GISeM 351 & Negro cónico & San Andrés Tlalnehuayocan, Ver. & $19^{\circ} 34^{\prime} ; 96^{\circ} 58^{\prime}$ & 1.640 & $351 \mathrm{nc}$ \\
\hline GISeM 263 & Castilla verde & Ixhuatlán del Café, Ver. & $19^{\circ} 04^{\prime} 10^{\prime \prime} ; 96^{\circ} 58^{\prime} 20^{\prime \prime}$ & 1.320 & $263 \mathrm{cv}$ \\
\hline GISeM 378 & Castilla verde & Ixtapa, Chis. & $16^{\circ} 48^{\prime} ; 92^{\circ} 54^{\prime}$ & 1.120 & $378 \mathrm{cv}$ \\
\hline GISeM 372 & Caldero & Cacahoatán, Chiapas, Chis. & $14^{\circ} 98^{\prime} ; 92^{\circ} 16^{\prime}$ & 486 & $372 \mathrm{cal}$ \\
\hline GISeM 375 & Caldero & Mixcum, Cacahoatán, Chis. & $15^{\circ} 01^{\prime} ; 92^{\circ} 13^{\prime}$ & 521 & $375 \mathrm{cal}$ \\
\hline GISeM 319 & Caldero & Unión Juárez, Chis. & $15^{\circ} 08^{\prime} ; 92^{\circ} 08^{\prime}$ & 2.220 & $319 \mathrm{cal}$ \\
\hline GISeM 327 & Negrito & El Rincón, Coscomatepec, Ver. & $19^{\circ} 05^{\prime} 00^{\prime \prime} ; 97^{\circ} 00^{\prime} 40^{\prime \prime}$ & 1.398 & $327 \mathrm{ne}$ \\
\hline GISeM 344 & Negrito & Tlaltengo, Coscomatepec, Ver. & $19^{\circ} 05^{\prime} 00^{\prime \prime} ; 97^{\circ} 00^{\prime} 40^{\prime \prime}$ & 1.480 & 344 ne \\
\hline GISeM 349 & Negrito & Rafael Lucio, Ver. & $19^{\circ} 35^{\prime} ; 96^{\circ} 59^{\prime}$ & 1.840 & 349 ne \\
\hline GISeM 350 & Negrito & San Andrés Tlalnehuayocan, Ver. & $19^{\circ} 34^{\prime} ; 96^{\circ} 58^{\prime}$ & 1.640 & $350 \mathrm{ne}$ \\
\hline GISeM 352 & Negrito & Teziutlán, Pue. & $19^{\circ} 49^{\prime} ; 97^{\circ} 21^{\prime}$ & 1.920 & 352 ne \\
\hline GISeM 367 & Verde espinoso & Texcoco, Méx. & $19^{\circ} 30^{\prime} ; 98^{\circ} 53^{\prime}$ & 2.340 & $367 \mathrm{ve}$ \\
\hline GISeM 342 & Negro xalapa & El Rincón, Coscomatepec, Ver. & $19^{\circ} 05^{\prime} 00^{\prime \prime} ; 97^{\circ} 00^{\prime} 40^{\prime \prime}$ & 1.398 & $342 n x$ \\
\hline GISeM 370 & Negro xalapa & Almolonga, Quetz., Guatemala & $14^{\circ} 82^{\prime} ; 91^{\circ} 48^{\prime}$ & 3.197 & $370 \mathrm{nx}$ \\
\hline GISeM 371 & Negro xalapa & Almolonga, Quetz, Guatemala & $14^{\circ} 82^{\prime} ; 91^{\circ} 48^{\prime}$ & 3.197 & $371 \mathrm{nx}$ \\
\hline GISeM 379 & Negro xalapa & Ixtapa, Chis. & $16^{\circ} 48^{\prime} ; 92^{\circ} 54^{\prime}$ & 1.120 & $379 \mathrm{nx}$ \\
\hline GISeM 386 & S. chinantlense & Valle Nacional, Oax. & $18^{\circ} 06^{\prime} ; 96^{\circ} 07^{\prime}$ & 57 & $386 \mathrm{sch}$ \\
\hline GISeM 377 & S. compositum & Talismán, Tuxtla Chico, Chis. & $14^{\circ} 96^{\prime} ; 92^{\circ} 15^{\prime}$ & 320 & $377 \mathrm{sc}$ \\
\hline GISeM 360 & Verde liso & Tlaltetela, Ver. & $19^{\circ} 19^{\prime} ; 96^{\circ} 54^{\prime}$ & 960 & $360 \mathrm{vl}$ \\
\hline GISeM 361 & Verde liso & Tlaltetela, Ver. & $19^{\circ} 19^{\prime} ; 96^{\circ} 54^{\prime}$ & 960 & $361 \mathrm{vl}$ \\
\hline GISeM 381 & Verde liso & Capoluca, Ver. & $18^{\circ} 48^{\prime} 10^{\prime \prime} ; 97^{\circ} 01^{\prime} 35^{\prime \prime}$ & 800 & $381 \mathrm{vl}$ \\
\hline GISeM 384 & Verde liso & Cartago, Costa Rica & $19^{\circ} 52^{\prime} ; 83^{\circ} 55^{\prime}$ & 1.435 & $384 \mathrm{vl}$ \\
\hline GISeM 353 & Verde espinoso & Teziutlán, Pue. & $19^{\circ} 49^{\prime} ; 97^{\circ} 21^{\prime}$ & 1.920 & $353 \mathrm{ve}$ \\
\hline GISeM 500 & Verde liso & Texcoco, Méx. & $19^{\circ} 30^{\prime} ; 98^{\circ} 53^{\prime}$ & 2.240 & $500 \mathrm{vl}$ \\
\hline
\end{tabular}
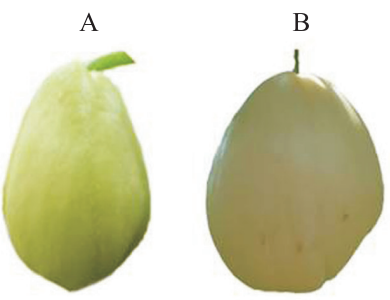

$\mathrm{H}$ I

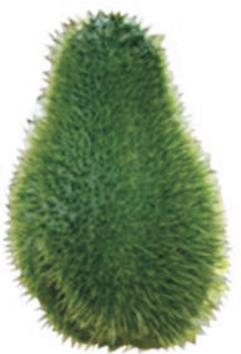

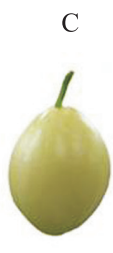

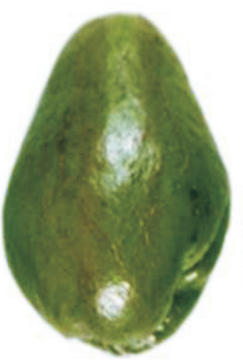

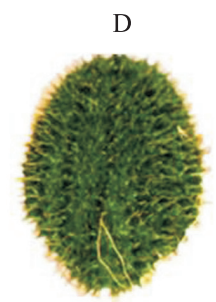

$\mathrm{K}$

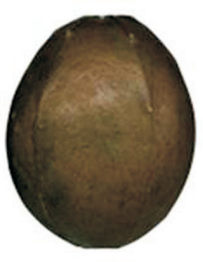

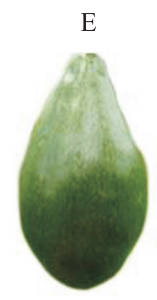

$\mathrm{L}$

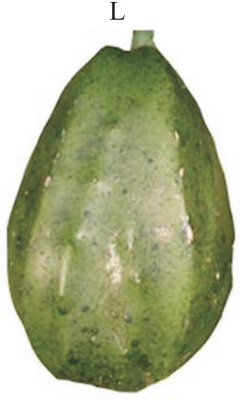

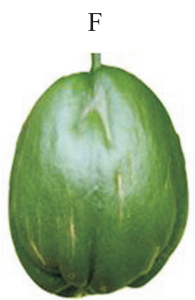

M

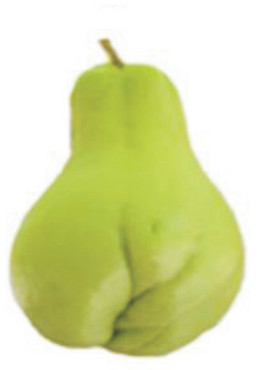

G

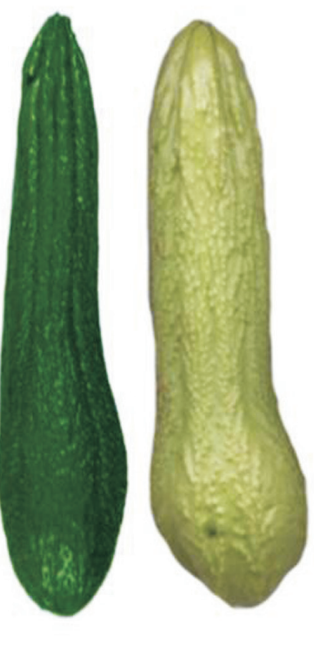

Figura 1. Accesiones de Sechium spp. utilizadas en el estudio: A, Cambray; B, Castilla blanco; C, Blanco pequeño; D, Sechium edule (silvestre); E, Negro cónico; F, Castilla verde; G, Caldero; H, Negrito; I, Verde espinoso; J, Negro xalapa; K, Sechium chinantlense (silvestre); L, Sechium compositum (silvestre); M, Verde liso. 
de bandeo en una lámpara luminosa de 15 W (Brune et al., 1998).

La variación genética fue estimada con base en diferentes parámetros que miden la estructura genética de la población, así como el grado de similitud y diversidad. Las estimaciones por accesión y por grupo varietal fueron: heterocigosidad observada (Ho) y heterocigosidad esperada $(\mathrm{He})$; número promedio de alelos por locus (NPAL), porcentaje de polimorfismo (PP), índice de Shannon (IS) e índice relativo de heterocigosidad (IRH), que permiten identificar el exceso o deficiencia de heterocigotes, en función del equilibrio de Hardy-Weinberg (Brown \& Weir, 1983; Ayala \& Kiger, 1984; Pasteur et al., 1988; Yeh, 1999). Fueron considerados polimórficos los loci con frecuencia del alelo más frecuente que no fuera superior al 95\%. El IRH se calculó de la forma siguiente: $\mathrm{IRH}=(\mathrm{Ho} / \mathrm{He})-1$, en que Ho es la heterocigosidad observada, y He es la heterocigosidad esperada.

Las medidas de diversidad descritas fueron estimadas con el programa genético Pogene (Yeh, 1999) para 23 loci isoenzimáticos, que presentaron el tipo de acción génica codominante. Se realizó, además, un análisis de componentes principales con las frecuencias génicas en 89 variables originales, mediante la matriz de covarianzas, con el procedimiento princomp de SAS (SAS Institute,1988); se estimaron con ello las distancias genéticas de Nei (1972) entre poblaciones, con las cuales se realizó un análisis de conglomerados de agrupación jerárquica, mediante el método de ligamiento promedio (UPGMA), a partir del cual se obtuvo el dendrograma correspondiente con el programa NTSYS (Rohlf, 1993).

\section{Resultados y Discusión}

De los 12 sistemas isoenzimáticos analizados, sólo el de peroxidasa no mostró polimorfismo; los loci monomórficos fueron Prx-2 y Prx-3. Las variables que explican en mayor proporción la variación observada se definieron con base en los vectores característicos de las variables originales con los componentes principales, con valores superiores a 0,15 . Los tres primeros componentes principales explicaron el 66,7\% de la varianza total (Cuadro 2). Los loci Cat-1, Est-2, Mdh-2, Me-1, Me-2, Pgd-2, Pgm-1, Pgm-3 y Rubisco, tuvieron una importante contribución en el CP1; el CP2 estuvo determinado por los loci Cat-2, Got, Mdh-1,
Me-3, Pgd-1, Sad-95 y Phi-1; y en el CP3 contribuyeron los loci Idh, Pgd1-100, Pgm2-95 y Sad-105.

En el cuadrante I de la Figura 2 se localizan accesiones provenientes principalmente de Chiapas, Guatemala y Veracruz, con forma y tamaño diferentes de fruto. En el cuadrante II, se agruparon accesiones sin espinas, provenientes de Veracruz, Chiapas y Costa Rica. En el cuadrante II y III, se agruparon accesiones principalmente por su origen - Veracruz -, más que por

Cuadro 2. Proporción de la varianza global, vectores y valores propios de los primeros tres componentes principales del análisis de componentes principales, para 30 accesiones de chayote (Sechium edule), con base en la matriz de covarianza.

\begin{tabular}{|c|c|c|c|}
\hline Locus $^{(1)}$ & CP1 & $\mathrm{CP} 2$ & $\mathrm{CP} 3$ \\
\hline Cat1-100 & $-0,100$ & 0,129 & 0,185 \\
\hline Cat1-105 & $-0,014$ & 0,022 & 0,008 \\
\hline Cat1-110 & $-0,025$ & 0,013 & $-0,077$ \\
\hline Cat1-115 & 0,139 & $-0,164$ & $-0,117$ \\
\hline Cat2-100 & $-0,176$ & 0,347 & $-0,088$ \\
\hline Cat2-115 & 0,185 & $-0,343$ & 0,089 \\
\hline Est2-100 & $-0,195$ & $-0,235$ & 0,193 \\
\hline Est2-115 & 0,195 & 0,235 & $-0,193$ \\
\hline Got-100 & $-0,140$ & 0,283 & 0,203 \\
\hline Idh-115 & 0,048 & 0,013 & 0,147 \\
\hline Mdh1-95 & 0,029 & $-0,023$ & $-0,021$ \\
\hline Mdh1-100 & $-0,041$ & $-0,094$ & 0,022 \\
\hline Mdh2-95 & 0,086 & $-0,036$ & 0,030 \\
\hline Mdh2-100 & $-0,102$ & 0,029 & $-0,070$ \\
\hline Me1-95 & $-0,011$ & $-0,018$ & 0,008 \\
\hline Me1-100 & $-0,167$ & $-0,131$ & 0,009 \\
\hline Me1-105 & $-0,080$ & $-0,036$ & 0,082 \\
\hline Me3-100 & $-0,027$ & 0,164 & $-0,004$ \\
\hline Me3-105 & $-0,032$ & $-0,098$ & $-0,048$ \\
\hline Pgd1-95 & 0,079 & 0,203 & 0,280 \\
\hline Pgd1-100 & $-0,055$ & $-0,173$ & $-0,271$ \\
\hline $\operatorname{Pgd} 2-100$ & $-0,171$ & $-0,154$ & $-0,034$ \\
\hline $\operatorname{Pgd} 2-105$ & $-0,040$ & $-0,033$ & 0,010 \\
\hline $\operatorname{Pgd} 2-115$ & 0,246 & 0,187 & $-0,065$ \\
\hline Pgm1-95 & $-0,018$ & 0,019 & $-0,029$ \\
\hline Pgm2-95 & $-0,036$ & 0,065 & $-0,098$ \\
\hline Pgm3-100 & $-0,221$ & 0,178 & $-0,318$ \\
\hline Rubisco-100 & $-0,148$ & 0,098 & 0,339 \\
\hline Rubisco-115 & 0,206 & $-0,090$ & $-0,285$ \\
\hline Sad-95 & $-0,002$ & 0,019 & $-0,022$ \\
\hline Sad-105 & 0,097 & $-0,005$ & 0,168 \\
\hline Phi1-95 & 0,007 & 0,075 & 0,070 \\
\hline Valor propio & 2,909 & 0,905 & 0,445 \\
\hline Variación explicada (\%) & 45,60 & 14,20 & 7,00 \\
\hline Variación acumulada (\%) & 45,60 & 59,80 & 66,70 \\
\hline
\end{tabular}

${ }^{(1)}$ Sólo se muestran los loci que definieron la variación en los CP1(componente principal 1), CP2 y CP3. 
forma y tamaño del fruto; en este grupo se encontraron chayotes semidomesticados como los Negritos y el Negro cónico. En el cuadrante III, también se observa otro grupo de origen veracruzano, con chayotes

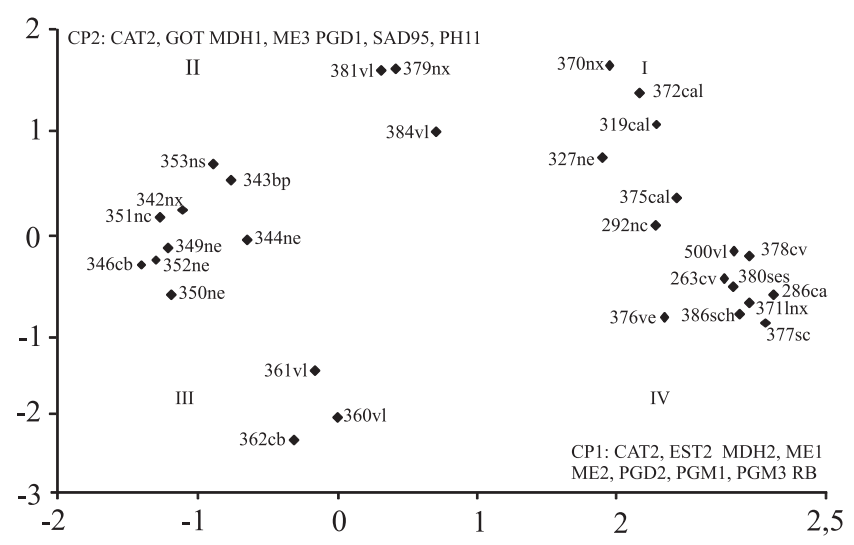

Figura 2. Dispersión de 30 accesiones de chayote, en función de los dos primeros componentes principales (CP), obtenidos con la matriz de covarianzas de 89 alelos isoenzimáticos. cuyos frutos no presentan espinas. En el cuadrante IV, se observan chayotes de diferente procedencia y, además, se agruparon los parientes silvestres $(S$. edule, $S$. chinantlense y $S$. compositum ) con diferentes tipos de chayotes; esta forma de agrupación probablemente se debe a que el chayote es una planta alógama $\mathrm{y}$, también, se debe a un posible flujo de genes entre poblaciones, sobre todo con $S$. edule.

El dendrograma, construido conforme Nei (1972), permitió la definición de seis grupos y seis subgrupos con base en una distancia genética de 0,24 (Figura 3). El grupo I se formó de nueve poblaciones de diferentes procedencias, en el cual se ubicaron los tipos silvestres (Ib) S. edule silvestre, S. compositum y $S$. chinantlense. Sechium chinantlense se caracteriza por ser de fruto piriforme aplanado y sin espinas, y es endémica de la región de la "chinantla Oaxaqueña", la cual se sitúa geográfica y climáticamente muy distante de los sitios de distribución de los otros tipos silvestres. Este grupo

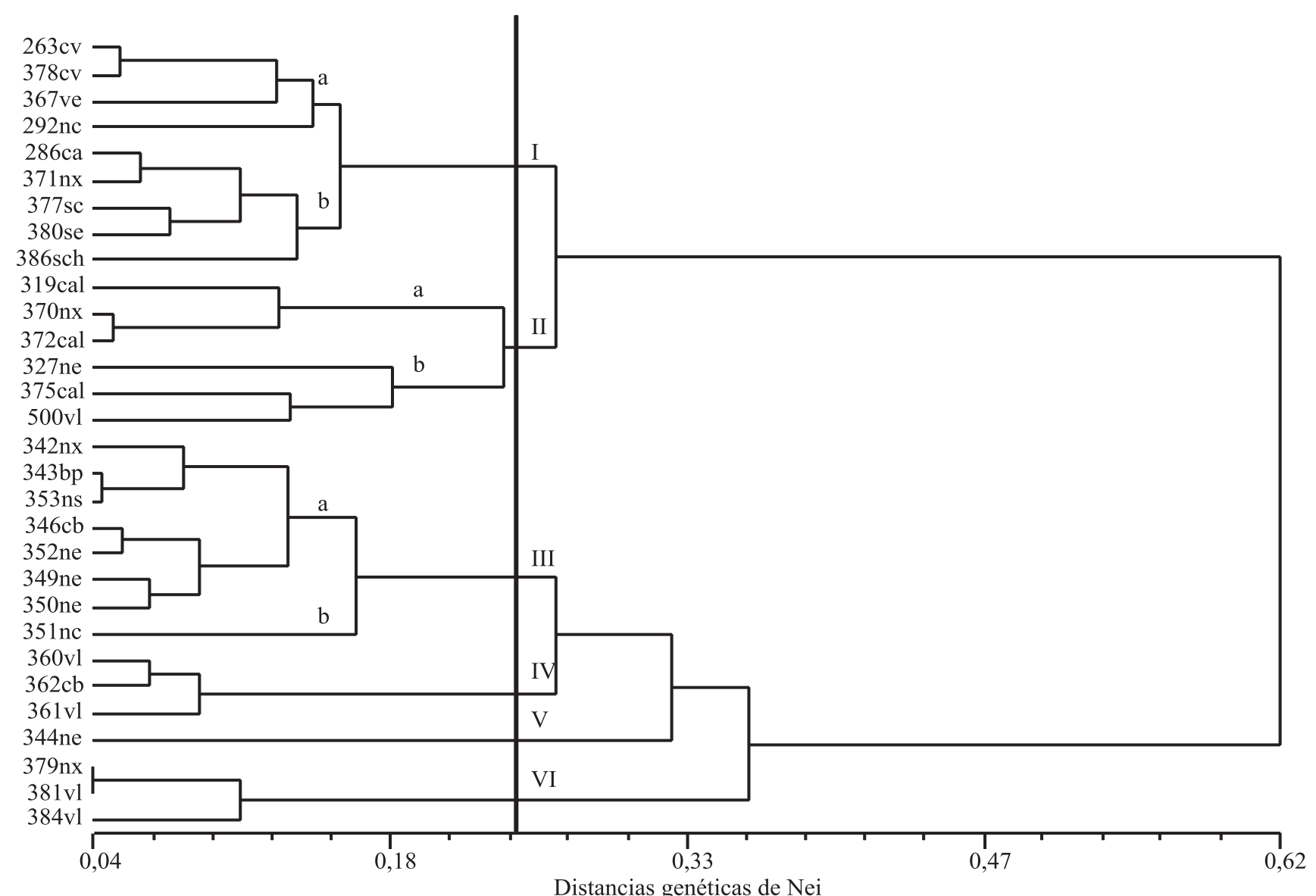

Figura 3. Dendrograma de agrupamiento de 30 accesiones de chayote, con base en 89 alelos isoenzimáticos. 
aglutinó, además, a diferentes variedades domesticadas de S. edule (Castilla verde, Verde liso, Cambray, Caldero y Verde espinoso), con características morfológicas como presencia o ausencia de espinas, forma y color del fruto. Resultados similares de agrupación de los tipos silvestres fueron reportados por Cross (2003), quien, en su análisis de la evolución taxonómica del género Sechium, registró la proximidad genética de $S$. compositum, S. chinantlense y $S$. edule, además de algunos tipos domesticados de este último, lo que fue atribuido a efectos de domesticación. En el subgrupo IIa, destacan dos poblaciones de Caldero y una de Negro xalapa (319nma, 370nx, 372nma), cuyos rasgos distintivos en sus frutos son: el color verde, forma alargada a muy alargada, liso y el tamaño de grande a muy grande. El subgrupo IIIa agrupó chayotes de tamaño pequeño (346al, 349nmi, 350nmi, $352 \mathrm{nmi}$ ), mientras que el IIIb ubicó exclusivamente una de las dos poblaciones de Negro cónico (351nc), una variedad encontrada raramente en huertos familiares, que presenta frutos de forma cónica. En el grupo IV, se observan chayotes sin espinas y de color amarillo (Castilla blanco) hasta verde oscuro (Negro xalapa), mientras que el grupo $\mathrm{V}$ se formó de una sola población (Negrito) y el cual no muestra cruzamiento con el resto de las variedades. En el grupo VI, se ubicaron las variedades genéticamente más distantes respecto a los tipos silvestres (Verde liso y Negro xalapa), las cuales son coincidentemente las de mayor cultivo extensivo y valor comercial dentro y fuera de México.
El número de alelos observados, de cada grupo varietal, osciló de 1,3 a 3, y el número promedio de alelos por locus fue 2,03 (Cuadro 3); los grupos silvestres fueron los de menor número de alelos comparados con los domesticados, como el Verde liso con tres alelos. Esto se puede atribuir a la manipulación y procedencia de los materiales y del tamaño de muestra que se utilizó, ya que Cambray, Castilla verde y silvestres tuvieron pocas muestras. De acuerdo con Hamrick et al. (1979), los valores obtenidos de alelos por locus son altos al compararlos con los que se reportan para cultivos de ciclo anual, como sería el caso del complejo de Sechium spp.

El número de loci polimórficos (NLP) osciló de 7 a 21 , y el promedio fue 13,7, con un porcentaje de polimorfismo entre los grupos varietales de 30,4 a $91,3 \%$ y promedio de $59,8 \%$ (Cuadro 3 ); este estimador de la diversidad indica que las poblaciones de chayote evaluadas conservan una amplia variación genética. En las accesiones de chayote mexicano, el polimorfismo es muy similar a lo reportado por Abdelnour \& Rocha (2008) con base en un estudio con diez sistemas isoenzimáticos, en 42 accesiones de chayote de Costa Rica, en las que encontraron $57,14 \%$ de polimorfismo. El porcentaje relativamente alto de polimorfismo puede ser el reflejo de la manipulación por el hombre, sobre todo por la dispersión a que ha sido sometido el chayote (Gottlieb, 1981). De acuerdo con Hamrick et al. (1979), los valores obtenidos de porcentaje de polimorfismo son altos, al compararlos con los valores para cultivos de ciclo anual. Cadena-Iñiguez (2005) reportó un porcentaje promedio de polimorfismo de

Cuadro 3. Heterocigosidad observada (Ho) y esperada (He), número de loci polimórficos (NLP); porcentaje de polimorfismo (PP); número de alelos por locus observados (NA) en complejo infraespecífico de Sechium spp.

\begin{tabular}{|c|c|c|c|c|c|c|}
\hline Grupo varietal & NLP & PP & NA & Ho & $\mathrm{He}$ & $\chi^{2}(\mathrm{Ho}: \mathrm{Ho}=\mathrm{He})$ \\
\hline Cambray & 8 & 34.78 & 1.47 & 0.019 & 0.156 & $*$ \\
\hline Castilla blanco & 16 & 69.57 & 2 & 0.053 & 0.258 & ns \\
\hline Blanco pequeño & 13 & 56.52 & 1.73 & 0.101 & 0.225 & ns \\
\hline Negro cónico & 18 & 78.26 & 2.34 & 0.038 & 0.372 & * \\
\hline Castilla Verde & 9 & 39.13 & 1.69 & 0.106 & 0.162 & ns \\
\hline Caldero & 13 & 56.52 & 2.17 & 0.019 & 0.269 & * \\
\hline Negrito & 21 & 91.30 & 2.95 & 0.075 & 0.372 & ns \\
\hline Verde espinoso & 18 & 78.26 & 2.21 & 0.033 & 0.358 & ns \\
\hline Negro xalapa & 19 & 82.61 & 2.69 & 0.064 & 0.396 & ns \\
\hline Verde liso & 20 & 86.96 & 3 & 0.053 & 0.389 & ns \\
\hline S. edule & 8 & 34.78 & 1.39 & 0.01 & 0.12 & * \\
\hline S. compositum & 9 & 39.13 & 1.48 & 0.08 & 0.13 & $\mathrm{~ns}$ \\
\hline S. chinantlense & 7 & 30.43 & 1.3 & 0.04 & 0.11 & ns \\
\hline Promedio & 13.77 & 59.87 & 2.03 & 0.05 & 0.26 & \\
\hline
\end{tabular}

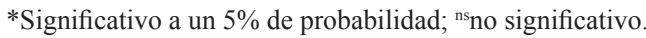


40,6\%, en 18 poblaciones de Sechium edule de ocho grupos varietales. Gwanama et al. (2000) encontraron, en Cucurbita moschata, $23,2 \%$ de polimorfismo mediante marcadores RAPD, mientras que Katzir et al. (2000) registraron hasta un $80 \%$ de polimorfismo en 28 accesiones de Cucurbita pepo con marcadores de ISSR; ambas especies con fuerte manipulación por el hombre.

Sin embargo, al comparar los valores de $\mathrm{He}$ y Ho, no se detectaron diferencias significativas con la prueba de $\chi^{2}$, excepto en Cambray, Negro cónico, Caldero y $S$. edule silvestre (Cuadro 3), lo que indica que en estos grupos varietales hay deficiencias de heterocigotos y, por lo tanto, no están en equilibrio Hardy-Weinberg, según la prueba de $\chi^{2}$. El IRH indica que en todos los grupos varietales hay una deficiencia de genotipos heterocigotos (Figura 4). Esta característica del complejo varietal de Sechium serviría para planificar una mejor estrategia de conservación in situ o en bancos de germoplasma. De manera previa a la conservación, sería necesario hacer que dichos grupos varietales entraran en equilibrio de Hardy-Weinberg, mediante apareamiento aleatorio, como lo sugiere Rincón Enríquez et al. (2005) para poblaciones silvestres de teozintle, al encontrar el mismo comportamiento.

De acuerdo al índice de Shannon (IS), el orden de diversidad en los grupos varietales de Sechium spp. es: Verde liso $>$ Negro xalapa y Negrito $>$ Negro cónico $>$ Verde espinoso $>$ Caldero $>$ Castilla blanco $>$ Blanco pequeño $>$ Castilla Verde $>$ Cambray $>S$. compositum

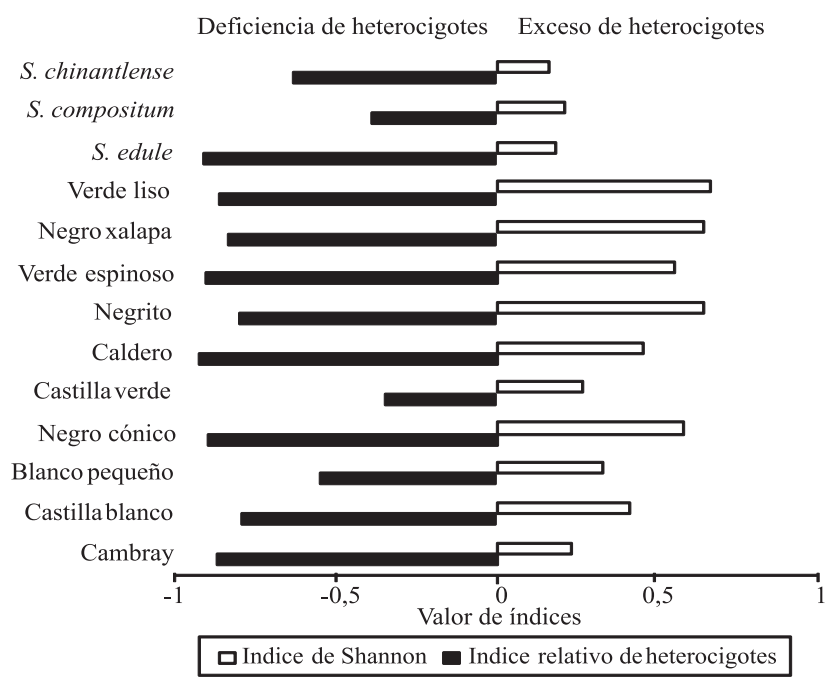

Figura 4. Índice relativo de heterocigosidad e índice de Shannon de 30 accesiones, de tres especies de Sechium spp. cultivados y silvestres, con base en 12 sistemas isoenzimáticos.
$>$ S. edule $>$ S. chinantlense (Figura 4). Para combinar ambas medidas de diversidad genética (PP y He), los valores de heterocigosidad esperada y porcentaje de loci polimórficos por grupo varietal están representados en la Figura 5. Las poblaciones de los grupos varietales con mayor variación fueron Negrito, Verde liso, Negro xalapa, Verde espinoso y Negro cónico; con variación intermedia fueron Castilla blanco, Caldero y Blanco pequeño; y con poca variación fueron Castilla verde, Cambray y los parientes silvestres. Este gradiente de variación coincidecon el observadomediante elíndicede Shannon, que indica que la amplia diversidad genética, en los grupos varietales Verde liso, Negro xalapa y Verde espinoso, puede ser atribuible a su dispersión a diferentes zonas agroecológicas de México por ser las variedades con mayor importancia comercial; este nivel de asociación ha sido reportado por Hamrick et al. (1979). También existen tendencias de formar grupos varietales por características fenotípicas distintivas, aun cuando el análisis de diversidad genética no lo muestre estrictamente, lo que puede deberse al efecto de las actividades humanas, como los usos, preferencias y prácticas culturales (Harlan, 1992).

Autores como Epperson \& Chung (2001) indican que una alta diversidad intrapoblacional puede constituir una respuesta adaptativa a la heterogeneidad espacial y temporal encontrada en las poblaciones. La mayoría de la variación en los caracteres adaptativos de las especies de Sechium es clinal (Cadena-Iñiguez, 2005) y se relaciona con gradientes ambientales, además de la manipulación, lo que podría resaltar el papel de los factores humano-ecológicos en la determinación,

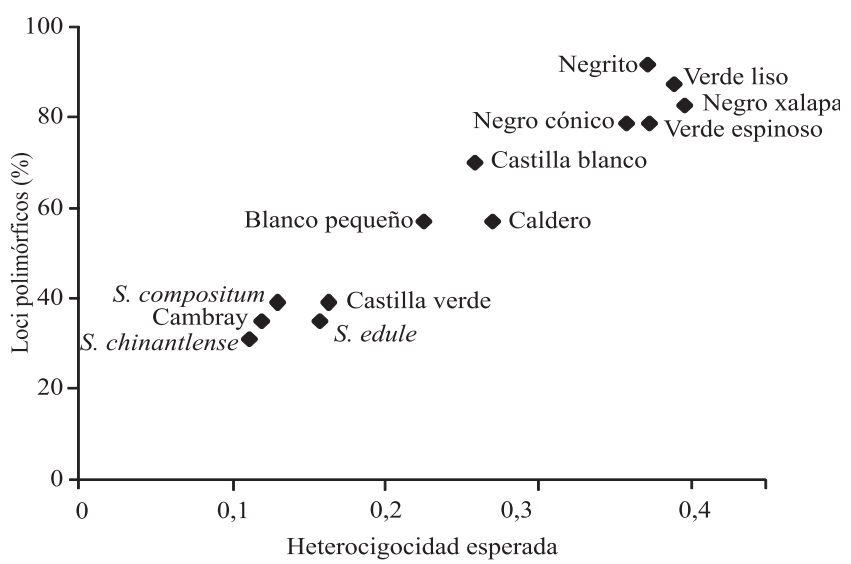

Figura 5. Variación genética de 30 poblaciones de tres especies de Sechium spp., cultivadas y silvestres, con base en 12 sistemas isoenzimáticos. 
extensión y distribución de la diversidad genética. Cadena-Iñiguez et al. (2008) sugieren que la variación fenotípica del complejo infraespecífico de $S$. edule está relacionada con cambios morfológicos y anatómicos y que los chayotes cultivados han seguido diferentes rutas, en el proceso co-evolutivo con el hombre y, con ello, la formación de grupos varietales, los mismos que se identifican fenotípicamente.

\section{Conclusiones}

1. Hay una amplia variación dentro del complejo infraespecífico e interespecífico de Sechium de México.

2. La diversidad genética de Sechium no es aleatoria, ya que se pueden distinguir los grupos varietales con base en el tamaño, color y ausencia o presencia de espinas de los frutos.

\section{Referencias}

ABDELNOUR, A.; ROCHA, O.J. Genetic characterization of a collection of chayote, Sechium edule (Jacq.) Swartz, in Costa Rica by using isozyme markers. Genetic Resources and Crop Evolution, v.55, p.163-170, 2008.

AVENDAÑO ARRAZATE, C.H.; CADENA IÑIGUEZ, J.; ARÉVALO GALARZA, M.L.C.; CAMPOS ROJAS, E.; CISNEROS SOLANO, V.M.; AGUIRRE MEDINA, J.F. Las variedades del chayote mexicano, recurso ancestral con potencial de comercialización. México: Grupo Interdisciplinario de Investigación en Sechium edule en México, A.C., 2010. 88p.

AYALA, F.J.; KIGER, J.A. Genética moderna. México: Fondo Educativo Interamericano, 1984. 836p.

BROWN, A.D.H.; WEIR, B.S. Measuring genetic variability in plant populations. In: TANKSLEY, S.D.; ORTON, T.J. (Ed.). Isozymes in plant genetics and breeding, part A. Amsterdam: Elsevier, 1983. p.219-240.

BRUNE, W.; ALFENAS, A.C.; GOES, T. Identificações específicas de enzimas em géis. In: ALFENAS, A.C. (Ed.). Eletroforese de isoenzimas e proteínas afins: fundamentos e aplicações em plantas e microorganismos. Viçosa: UFV, 1998. p.201-317.

CADENA-IÑIGUEZ, J.; ARÉVALO-GALARZA, L.; AVENDAÑO-AZZARATE， C.H.; SOTO-HERNÁNDEZ， M.; RUIZ-POSADAS, L.M.; SANTIAGO, O.E.; ACOSTA, R.M.; CISNEROS-SOLANO, V.M.; AGUIRRE-MEDINA, J.F.; OCHOA, M.D. Production, genetics and postharvest management of Sechium edule (Jacq.) Sw. Invited review. Fresh Produce Journal, v.1, p.41-53, 2007.

CADENA-IÑIGUEZ, J.; AVENDAÑO-AZZARATE, C.H.; SOTO-HERNÁNDEZ, M.; RUIZ-POSADAS, L.M.; AGUIRRE-MEDINA, J.F; ARÉVALO-GALARZA, L. Infraspecific variation of Sechium edule (Jacq.) Sw. in the state of
Veracruz, Mexico. Genetic Resources and Crop Evolution, v.55, p.835-847, 2008.

CADENA-IÑIGUEZ, J. Caracterización morfoestructural, fisiológica, química y genética de diferentes tipos de chayote (Sechium edule (Jacq.) Sw.). 2005. 156p. Tesis (Doctorado) Colegio de Postgraduados, Texcoco.

CADENA-IÑIGUEZ, J.; RUIZ-POSADAS, L.M.; TREJO-LÓPEZ, C.; SÁNCHEZ-GARCÍA， P.; AGUIRRE-MEDINA， J.F. Intercambio de gases y relaciones hídricas del chayote (Sechium edule (Jacq.) Sw. Revista Chapingo. Serie Horticultura, v.7, p.21-35, 2001.

CROSS, H. Evolution, systematics, and domestication in Sechium and related genera (Sicyeae, Cucurbitaceae). Columbia: Columbia University, 2003. 364p.

ENGELS, J.M.M. Variation in Sechium edule in Central America. Journal of the American Society of Horticultural Science, v.108, p.706-710, 1983.

EPPERSON, B.K.; CHUNG, G.I.M. Spatial genetic structure of allozyme polymorphisms within populations of Pinus strobus (Pinaceae). American Journal of Botany, v.88, p.1006-1010, 2001.

FLORES, E.M. El chayote, Sechium edule Swartz (Cucurbitaceae). Revista Biología Tropical, v.37, p.1-54, 1989.

FONT, Q.P. Diccionario de botánica. Barcelona: Labor, 1979. 1090p.

GOTTLIEB, L.D. Electrophoretic evidence and plant populations. Progress in Phytochemistry, v.7, p.1-46, 1981.

GWANAMA, C.; LABUSCHAGNE, M.T.; BOTHA, A.M. Analysis of genetic variation in Cucurbita moschata by random amplified polymorphic DNA (RAPD) markers. Euphytica, v.113, p.19-24, 2000.

HAMRICK, J.L.; LINHART, Y.B.; MITTON, J.B. Relationships between life history characteristics and electrophoretically detectable genetic variation in plants. Annual Review of Ecology and Systematics, v.10, p.173-200, 1979.

HARLAN, J.R. Crops and man. Madison: American Society of Agronomy: Crop Science Society of America, 1992. 284p.

JACKSON, F. The coevolutionary relationship of humans and domesticated plants. Yearbook of Physical Anthropology, v.39, p.161-176, 1996.

KATZIR, N.; TADMOR, Y.; TZURI, G.; LESHZESHEN, E.; MOZES-DAUBE, N.; DANIN-POLEG, Y.; PARIS, H.S. Further ISSR and preliminary SSR analysis of relationships among accessions of Cucurbita pepo. Acta Horticulturae, v.510, p.433-440, 2000.

LARA-SAADE, R. Chayote: Sechium edule (Jacq.) Sw. Rome: International Plant Genetic Resources Institute, 1996. 57p. Promoting the conservation and use of underutilized and neglected crops, 8).

MAFFIOLI, A. Recursos genéticos de Sechium edule (Jacq.) Swartz (Cucurbitacea). Turrialba: CATIE, 1981. 151p.

NEI, M. Genetic distance between populations. American Naturalist, v.106, p.283-292, 1972.

NEWSTROM, L.E. Evidence for the origin of chayote Sechium edule (Cucurbitaceae). Economic Botany, v.45, p.410-428, 1991. 
PASTEUR, N.; PASTEUR, G.; BONHOMME, F.; CATALAN, J.; BRITTON-DAVIDIAN, J. Practical isozyme genetics. Chichester: Ellis Horwood Limited, 1988. 215p.

RINCÓN ENRÍQUEZ, G.; RAMÍREZ VALLEJO, P.; SÁNCHEZ GONZÁLEZ, J.J.; YAMAKAKE, T.A.K. 2005. Variación isoenzimática en poblaciones de teocintle. Revista Fitotecnia Mexicana, v.28, p.105-113, 2005.

ROHLF, J.F. NTSYS-pc: numerical taxonomy and multivariate analysis system. Version 1.8. New York: Stony Brook, 1993.
SAS INSTITUTE. SAS/STAT: user's guide. Version $6.4^{\text {th }}$ ed. Cary: SAS Institute, 1988.

STUBERT, C.W.; GOODMAN, M.M. Techniques and scoring procedures for starch gel electrophoresis of enzymes from maize (Zea mays L.). Raleigh: North Carolina State University, 1988. 87p.

YEH, C.F. POPGENE version 1.31: Microsoft window-based freeware for population genetic analysis: quick user guide. Edmonton: University of Alberta, 1999.

Recibido en 12 de octubre de 2011 y aprobado en 30 de enero de 2012 